H. UEDA

KODAI MATH J.

3 (1980), 212-223

\title{
UNICITY THEOREMS FOR ENTIRE FUNCTIONS
}

\author{
By HidehaRU UEDA
}

1. Ozawa has proved some unicity theorems for entire functions in [7]. His main interest lies in the following problem: How does the distribution of zero-one sets affect the unicity in the case of entire functions? In this paper we shall be concerned with the same problem.

If two entire functions $f$ and $g$ have the same $a$-points with the same multiplicities, we denote this by $f=a \rightleftarrows g=a$ for simplicity's sake. And we denote the order of $f$ by $\rho_{f}$.

With this notation, we may state two unicity theorems in [7] as in the following manner.

Theorem A. ([7, Theorem 4]) Let $f$ and $g$ be entire functions. Assume that $\rho_{f}, \rho_{g}<\infty, f=0 \rightleftarrows g=0, f=1 \rightleftarrows g=1$ and $\delta(0, f)>1 / 2$. Then $f g \equiv 1$ unless $f \equiv g$.

Theorem B. (7, Theorem 7]) Let $f$ and $g$ be entrre functions. Assume that $f=1 \rightleftarrows g=1, \delta(0, f)>0$ and 0 is lacunary for $g$. Then $f g \equiv 1$ unless $f \equiv g$.

In this paper we shall give four results on unicity which are related to Theorem A and one depending on Theorem B. The detail is as follows.

As Ozawa pointed out in [7], the assumption " $\delta(0, f)>1 / 2$ " in Theorem A is best possible. Hence some additional conditions are needed in the case of $\delta(0, f) \leqq 1 / 2$ in order to obtain the conclusion " $f \equiv g$ ". With respect to this point we prove the following two theorems.

THEOREM 1. Let $f$ and $g$ be entire functions. Assume that $\rho_{f}, \rho_{g}<\infty$, and (A) $f=0 \rightleftarrows g=0, f=1 \rightleftarrows g=1,(\mathrm{~B}) 0<\delta(0, f) \leqq 1 / 2$ and further that (C) there exists at least one zero $w$ such that $f^{(n)}(w)=g^{(n)}(w) \neq 0$ and $f^{(j)}(w)=g^{(j)}(w)=0$ for $j=0,1, \cdots, n-1$. Then $f \equiv g$.

THEOREM 2. Let $f$ and $g$ be entrre functions. Assume that $\rho_{f}, \rho_{g}<\infty$, and (A) $f=0 \rightleftarrows g=0, f=1 \rightleftarrows g=1,(\mathrm{~B})^{\prime} \delta(0, f)=0$. Further assume that (C) there exist infinitely many zeros $\{w\}$ of $f$ for which the same condition is satısfied as $w$ in (C) of Theorem 1 . Then $f \equiv g$.

In the above Theorem 2, we cannot replace "infinitely many zeros" by "finitely many zeros". To see this, we may consider the following example.

Received April 10, 1979 
Let $f$ and $g$ be as follows:

$$
f(z)=\frac{1-e^{2 \pi i L(z)}}{1-e^{2 \pi i z}}, g(z)=\frac{1-e^{2 \pi i L(z)}}{1-e^{2 \pi \imath z}} e^{2 \pi i[z-L(z)]},
$$

where

$$
L(z)=(n+1) ! \int_{0}^{z} \prod_{k=1}^{n}(t-k) d t \quad(n=1,2, \cdots) .
$$

Then $f$ and $g$ satisfy the conditions (A) and (B)' in Theorem 2. However, there exist only $n$ zeros: $\{k\}_{k=1}^{n}$ of $f$ for which the same condition is satisfied as $w$ in (C) of Theorem 1.

Now it is natural to ask whether the order restriction of $f$ and $g$ in Theorem $A$ can be removed or not. In this direction, we have the following fact:

THEOREM 3. Let $f$ and $g$ be entrre functions. Assume that (A) $f=0 \rightleftarrows g=0$, $f=1 \rightleftarrows g=1$ and $(C) \tilde{N}(r, f, g)<k[m(R, f)+m(r, g)](0<k<1 / 6)$, where $\tilde{N}(r, f, g)$ is defined as follows:

$$
\begin{gathered}
\tilde{N}(r, f, g)=\int_{0}^{r} \frac{\tilde{n}(t, f, g)-\tilde{n}(0, f, g)}{t} d t+\tilde{n}(0, f, g) \log r, \\
\tilde{n}(r, f, g)=\sharp\left\{w \in\{|z| \leqq r\} ; \begin{array}{l}
f^{(j)}(w)=g^{(j)}(w)=0 \quad(\jmath=0,1, \cdots, m-1) \\
f^{(m)}(w) \neq g^{(m)}(w), f^{(m)}(w) \cdot g^{(m)}(w) \neq 0, \quad m>0
\end{array}\right\} .
\end{gathered}
$$

Then $f g \equiv 1$ unless $f \equiv g$.

From Theorem 3 we deduce the following two corollaries immediately.

COROllary 1. Let $f$ and $g$ be entrre functions. Assume that $\rho_{f}=\rho_{g}=\infty$, $f=0 \rightleftarrows g=0, f=1 \rightleftarrows g=1$ and $\delta(0, f)>5 / 6$. Then $f g \equiv 1$ unless $f \equiv g$.

COROLlary 2. Let $f$ and $g$ be entrre functions. Assume that $\rho_{f}=\rho_{g}=\infty$, $f=0 \rightleftarrows g=0$ and $f=1 \rightleftarrows g=1$. Further assume that all zero-points excepting at most finte number have multıplicities $\geqq 7$. Then $f g \equiv 1$ unless $f \equiv g$.

Thirdly as another application of the proof of Theorem A, we have

THEOREM 4. Let $f$ and $g$ be entrre functions. Assume that $\rho_{f}, \rho_{g}<\infty, f \neq g$, and (A) $f=0 \rightleftarrows g=0, f=1 \rightleftarrows g=1$ and that (B) $f$ has at least one zero and all of them lie on the real axis. Then $f$ and $g$ are one of the following two forms:

1) $f(z)=A\left[e^{\imath(a z+b)}-e^{\imath d}\right], \quad g(z)=-\frac{f(z)}{(1-c) f(z)+c} \quad(c \neq 0,1)$,

$$
A=\left|\frac{c}{1-c}\right|, \quad a(\neq 0), b, d: \text { real constants }
$$


2) $f(z)=\frac{1-e^{\imath m(a z+b)}}{1-e^{\imath(a z+b)}}, \quad g(z)=\frac{e^{-\imath(m-1)(a z+b)}-e^{\imath(a z+b)}}{1-e^{\imath(a z+b)}}$, $a(\neq 0), b$ : real constants, $m(|m| \geqq 2)$ : an integer.

This theorem yields the following fact immediately.

COROLlaRY 3. Let $f$ and $g$ satisfy the assumptions (A), (B)' and $\rho_{f}, \rho_{g}<\infty$ of Theorem 3. Further assume enther (i) $f=1$ has at most one root, or (ii) not all the roots of $f=1$ lie on a stranght line being parallel to the real axis. Then $f \equiv g$.

Finally as an application of Theorem B, we prove the following

THEOREM 5. Let $f$ and $g$ be entrre functions. Assume that $\rho_{f}, \rho_{g}<\infty$, and (A) $g \neq 0, f(0)=1, f=1 \rightleftarrows g=1$ and that $(\mathrm{B})^{\prime}$ the roots of $f=0$ exist and all of them lie on the real axis. Then $f$ and $g$ are one of the following three forms:

1) $f(z)=(1 / 2+i b)+(1 / 2-i b) e^{\imath a z}, \quad g(z)=e^{\imath a z}$,

$$
a(\neq 0), b \text { : real constants, }
$$

2) $f(z)=1-e^{\imath a z}+e^{2 \imath a z}, \quad g(z)=e^{\imath a z}$,

$a(\neq 0)$; a real constant,

3) $f(z)=1+i b e^{-\imath a z}-i b e^{\imath a z}(=1+2 b \sin (a z)), \quad g(z)=e^{2 \imath a z}$, $a(\neq 0), b(|b| \geqq 1 / 2)$ : real constants .

2. In order to prove our theorems, we need the following several lemmas.

Lemma 1. ([1, Proposition 1]) Let $\mathcal{A}$ be the class of all enture functions having only real zeros and real ones. If $f$ is not a real entire function, then it is necessarily one of the following two forms:

(i) $f(z)=\frac{\sin (\xi z+\eta) e^{2\left(\xi z+\eta_{1}\right)}}{\sin \left(\eta-\eta_{1}\right)}, \quad\left(\sin \left(\eta-\eta_{1}\right) \neq 0\right)$

$\xi, \eta, \eta_{1}:$ real constants,

(ii) $f(z)=\frac{\sin \rho(\xi z+\eta) e^{\imath(p-1)(\xi z+\eta)}}{\sin (\xi z+\eta)}$,

$\xi, \eta$ : real constants, $p(\neq 0,1):$ an integer.

Lemma 2. (c.f. [3, Theorem 1.11]) Let $P(z)$ be a canonical product of genus q. Then $m(r, P)=o\left(r^{q+1}\right)(r \rightarrow \infty)$.

LEMma 3. ([2, Theorem 1., Corollary 1.]) Let $f$ be an entire function of finite order, all of whose zeros lie on the real axis. If the genus $q$ of $f$ is not 
less than two, then $\delta(0, f)>0$. Further if all the zeros of $f$ lie on the positwe (or negative) real axis, then $\delta(0, f)>0$ unless $q=0$.

Lemma 4. (c.f.[3, Theorem 2.1.]) Assume that $f$ is an entrre function. Let $a_{1}, \cdots, a_{p}(p \geqq 2)$ be distinct finte complex numbers. Then

$$
(p-1) m(r, f)+N_{1}(r) \leqq \sum_{\nu=1}^{p} N\left(r, a_{\nu}, f\right)+S(r, f),
$$

where $S(r, f)=O(\log r \cdot m(r, f))$ as $r \rightarrow \infty$ through all values if $\rho_{r}<\infty$, and as $r \rightarrow \infty$ outside a set $E$ of finte linear measure otherwise.

LEMma 5. ([5, Theorem 4.]) Let $f_{n}(n=1, \cdots, p)$ be non-constant enture functions satisfying $\sum_{n=1}^{p} \alpha_{n} f_{n}=1$ with non-zero constants $\alpha_{n}$. Then

$$
\sum_{n=1}^{p} \delta\left(0, f_{n}\right) \leqq p-1
$$

3. Proof of Theorems 1 and 2. We refer to Ozawa's argument in [7, p. 416-p. 419]. Firstly, from $\rho_{f}, \rho_{g}<\infty$ and the assumption (A), we have

$$
f=g e^{\alpha}, \quad f-1=(g-1) e^{\beta}
$$

with polynomials $\alpha$ and $\beta$. Next, we divide our argument into the following three cases in accordance with Ozawa's.

Case $1 . \quad e^{\beta} \equiv a$ constant $c(\neq 0)$

Case 2. $e^{\alpha-\beta} \equiv a$ constant $c(\neq 0)$

Case 3. $e^{\alpha} \neq \equiv a$ constant and $e^{\alpha-\beta} \neq \equiv$ constant

Now we start from the Case 1 . In this case $f-1=c(g-1)$. Since $f$ has zeros, $c$ must be one. This implies $f \equiv g$. Next we consider the Case 2. In this case $f=c e^{\beta} g(c \neq 0), f-1=e^{\beta}(g-1)$. If $c=1$, we have $f \equiv g$. If $c \neq 1$, we have

$$
g=\underset{(1-c) f+c}{f} \quad(c \neq 0,1) .
$$

Since $g$ is an entıre function, $(1-c) f+c=0$ has no roots. This implies $\delta\left(-\frac{c}{1-c}, f\right)=1$, so we have $\delta(0, f)=0$. Further from the fact that $(1-c) f+c$ $\neq 0$, we see the existance of a polynomial $P(z)$ satisfying $(1-c) f+c=e^{P(z)}$. Denote an arbitrary zero of $f$ by $u$. If we expand $f$ and $g$ in power series around the point $z=u$, we obtain

$$
f(z)=\frac{e^{P(z)}-c}{1-c}=\frac{c}{1-c} \frac{P^{(m)}(u)}{m !}(z-u)^{m}+\cdots .
$$

and 


$$
g(z)=\frac{e^{P^{(z)}}-c}{1-c} e^{-P(z)}=\frac{1}{1-c} \frac{P^{(m)}(u)}{m !}(z-u)^{m}+\cdots \quad(c \neq 0,1),
$$

where $m$ is the least positive integer for which $H^{(n)}(u)$ does not vanish. These expansions show that the assumption $(C)^{\prime}$ in Theorem 2 is not satisfied. Finally, we consider the Case 3. In this case, (3.1) implies

$$
f=\frac{1-e^{\beta}}{1-e^{\beta-\alpha}}, \quad g=\frac{1-e^{\beta}}{1-e^{\beta-\alpha}} e^{-\alpha} .
$$

Since $f$ is an entire function, we deduce from (3.2)

$$
\beta(z)=2 \pi \imath L(y(z)), \quad 2 \pi \imath y(z)=\beta(z)-\alpha(z)
$$

with a polynomial $L(z)$ of degree $\geqq 1$ (See [7, p. 417]).

Assume first that the degree of $L(z)$ is greater than one. Then Ozawa's argument in $[7$, p. 417, p. 418$]$ show $\delta(0, f)=0$. Denote an arbitrary zero of $f$ which satisfies the condition described in (C) of Theorem 1 by $u$. It is easy to see from (3.3) that such $w$ satisfies $e^{2 \pi \imath y(w)}=1$. Since $w$ is a zero of $f$, the order of 1-point of $e^{2 \pi i L(y(z))}$ at $z=w$ is higher than that of $e^{2 \pi i y(z)}$ at $z=w$. This implies that the order of 1-point of $e^{2 \pi i L(y(z))}$ at $z=w$ is at least two. If we put $H(z)=1-e^{2 \pi i L(y(z))}$, we have $H^{\prime}(w)=-2 \pi \imath L^{\prime}(y(w)) y^{\prime}(w)=0$. However, since $L$ and $y$ are polynomials in $z$, the number of such $w$ 's is at most finite. Thus the assumption $(C)^{\prime}$ is not satisfied.

Assume next that the degree of $L(z)$ is one. Then $f$ and $g$ are following forms: (see $[7$, p. 418])

$$
f(z)=\frac{1-e^{2 \pi \imath m y(z)}}{1-e^{2 \pi i y(z)}}, \quad g(z)=\frac{1-e^{2 \pi \imath m y(z)}}{1-e^{2 \pi \imath y(z)}} e^{2 \pi i(1-m) y(z)} \quad(m: \text { an integer }) .
$$

Suppose that $m \neq-1,0,1$. This case can be handled quite similarly as the above case, so that it is shown that there are no points $\{w\}$ satisfying the condition described in (C) of Theorem 1. Assume that $m=0$. Then $f \equiv g \equiv 0$. Assume that $m=1$. Then $f \equiv g \equiv 1$. Assume that $m=-1$. Then $f=-e^{-\left(m-2 y^{\prime} z\right)}$. This is absurd.

This completes the proof.

4. Proof of Theorem 3. By assumption (A), there exist two entire functions $\alpha$ and $\beta$ such that

$$
f=e^{\alpha} g, \quad f-1=(g-1) e^{3} .
$$

Firstly, assume either $e^{\beta}$ or $e^{\alpha-\beta}$ is a constant $c(\neq 0)$. In such cases Ozawa's argument in [7, p. 416, p. 417] and simple computations in the proof of Theorems 1 and 2 show that $c$ must be one, otherwise the assumption (C) of Theorem 3 is not satisfied. This implies $f \equiv g$.

Next, assume neither $e^{\beta}$ nor $e^{\alpha-\beta}$ are constants. In view of (4.1), we have 


$$
f=\frac{1-e^{\beta}}{1-e^{\beta-\alpha}}, \quad g=\frac{1-e^{\beta}}{1-e^{\beta-\alpha}} e^{-\alpha} .
$$

Since $f$ is an entire function, (4.2) implies

$$
\begin{aligned}
0 \leqq N(r, 0, f) & =n\left(r, 1, e^{\beta}\right)-N\left(r, 1, e^{\beta-\alpha}\right) \\
& =N_{1}\left(r, 1, e^{\beta}\right)+\bar{N}\left(r, 1, e^{\beta}\right)-N\left(r, 1, e^{\beta-\alpha}\right) \\
& \leqq N_{1}\left(r, 1, e^{\beta}\right)+\tilde{N}(r, f, g),
\end{aligned}
$$

where used the following fact:

$$
\tilde{N}(r, f, g)=\bar{N}\left(r, 1, e^{\beta}\right)-\bar{N}\left(r, 1, e^{\beta-\alpha}\right) .
$$

To see this, it is sufficient to prove

$$
\tilde{n}(r, f, g)=\bar{n}\left(r, 1, e^{\beta}\right)-\bar{n}\left(r, 1, e^{\hat{\gamma}-\alpha}\right) .
$$

However, (4.4) is an immediate consequence of (4.2).

Combining (4.3) and the assumption (C)", we have

$$
N(r, 0, f) \leqq N_{1}\left(r, 1, e^{\beta}\right)+k[m(r, f)+m(r, g)] .
$$

To estimate the right hand side of (4.5), we proceed as follows. Applying Lemma 4 with $f=e^{\beta}$, we have

$$
\begin{aligned}
m\left(r, e^{\beta}\right)+N_{1}\left(r, e^{\beta}\right) & \leqq N\left(r, 1, e^{\beta}\right)+O\left(\log r \cdot m\left(r, e^{\beta}\right)\right) \quad\left(r \notin E\left(e^{\beta}\right), r \rightarrow \infty\right) \\
& \leqq m\left(r, e^{\beta}\right)+o\left(\log r \cdot m\left(r, e^{\beta}\right)\right) .
\end{aligned}
$$

This yields that

$$
N_{1}\left(r, 1, e^{\beta}\right) \leqq N_{1}\left(r, e^{\beta}\right)=O\left(\log r \cdot m\left(r, e^{\beta}\right)\right) \quad\left(r \notin E\left(e^{\beta}\right), r \rightarrow \infty\right) .
$$

Also (4.6) implies that

$$
\lim _{\substack{r \rightarrow \infty \\ r \in\left(e^{\beta}\right)}} \frac{N\left(r, 1, e^{\beta}\right)}{m\left(r, e^{\beta}\right)}=1 .
$$

In the same way we have

$$
\lim _{\substack{r \rightarrow \infty \\ r \cdot E\left(e^{\beta-\alpha}\right)}} \frac{N\left(r, 1, e^{\beta-\alpha}\right)}{m\left(r, e^{\beta-\alpha}\right)}=1 .
$$

It follows from (4.3), (4.8) and (4.9) that

$$
m\left(r, e^{\beta}\right) \geqq(1-o(1)) m\left(r, e^{\beta-\alpha}\right) \quad\left(r \notin E\left(e^{\beta}\right) \cup E\left(E^{\beta-\alpha}\right) \equiv E^{\prime}, r \rightarrow \infty\right) .
$$

Hence evidently we have

$$
m(r, f) \leqq m\left(r, e^{\beta}\right)+m\left(r, e^{\beta-\alpha}\right)+o(1) \leqq(2+o(1)) m\left(r, e^{\beta}\right) \quad\left(r \notin E^{\prime}, r \rightarrow \infty\right),
$$

and so,

$$
\begin{aligned}
m(r, g) & \leqq m(r, f)+m\left(r, e^{n}\right) \leqq m(r, f)+m\left(r, e^{\beta}\right)+m\left(r, e^{\beta-r}\right) \\
& \leqq(4+o(1)) m\left(r, e^{\beta}\right) \quad\left(r \notin E^{\prime}, r \rightarrow \infty\right) .
\end{aligned}
$$


Substituting (4.7), (4.11) and (4.12) into (4.5), we obtain

$$
N(r, 0, f)<(6 k+o(1)) m\left(r, e^{\beta}\right)+O(\log r) \quad\left(r \in E^{\prime}, r \rightarrow \infty\right) .
$$

To complete the proof, we may use a method of Nevanlinna [4]. If we put $\varphi_{1}=e^{\beta}, \varphi_{2}=-e^{\beta-\alpha} \cdot f$, and $\varphi_{3}=f$, then (4.2) implies $\varphi_{1}+\varphi_{2}+\varphi_{3} \equiv 1$, so that $\varphi_{1}^{(n)}+\varphi_{2}^{(n)}+\varphi_{3}^{(n)} \equiv 0(n=1,2)$. Further we put

$$
\Delta \equiv\left|\begin{array}{ccc}
1 & 1 & 1 \\
\varphi_{1}^{\prime} / \varphi_{1} & \varphi_{2}^{\prime} / \varphi_{2} & \varphi_{3}^{\prime} / \varphi_{3} \\
\varphi_{1}^{\prime \prime} / \varphi_{1} & \varphi_{2}^{\prime \prime} / \varphi_{2} & \varphi_{3}^{\prime \prime} / \varphi_{3}
\end{array}\right| .
$$

Assume first that $\Delta \not \equiv 0$. Then

$$
\varphi_{1}=\frac{\Delta^{\prime}}{\Delta}, \quad \Delta^{\prime} \equiv\left|\begin{array}{ll}
\varphi_{2}^{\prime} / \varphi_{2} & \varphi_{3}^{\prime} / \varphi_{3} \\
\varphi_{2}^{\prime \prime} / \varphi_{2} & \varphi_{3}^{\prime \prime} / \varphi_{3}
\end{array}\right|
$$

Direct computation shows that

$$
\begin{aligned}
\Delta= & \frac{f^{\prime \prime}}{f}\left(\beta^{\prime}-\alpha^{\prime}\right)-2\left(\frac{f^{\prime}}{f}\right)^{2}\left(\beta^{\prime}-\alpha^{\prime}\right)+\frac{f^{\prime}}{f}\left[\left(\beta^{\prime}\right)^{2}-\left(\alpha^{\prime}\right)^{2}-2\left(\beta^{\prime}-\alpha^{\prime}\right)-\left(\beta^{\prime \prime}-\alpha^{\prime \prime}\right)\right] \\
& +\beta^{\prime}\left(\beta^{\prime \prime}-\alpha^{\prime \prime}\right)+\beta^{\prime}\left(\beta^{\prime}-\alpha^{\prime}\right)-\left(\beta^{\prime}-\alpha^{\prime}\right)\left[\beta^{\prime \prime}+\left(\beta^{\prime}\right)^{2}\right] .
\end{aligned}
$$

From this we easily obtain $N(r, \Delta) \leqq N(r, 0, f)$. Combıning this and (4.13) we have

$$
N(r, \Delta) \leqq(6 k+o(1)) m\left(r, e^{\beta}\right)+O(\log r) \quad\left(r \notin E^{\prime}, r \rightarrow \infty\right) .
$$

On the other hand, from (4.15) we have

$$
\begin{aligned}
m\left(r, e^{\beta}\right) & =m\left(r, \varphi_{1}\right)=m\left(r, \Delta^{\prime} / \Delta\right) \leqq m\left(r, \Delta^{\prime}\right)+m\left(r, \Delta^{-1}\right) \\
& \leqq m\left(r, \Delta^{\prime}\right)+m(r, \Delta)+N(r, \Delta) .
\end{aligned}
$$

Further, we have from (4.10) and (4.11)

$$
\begin{aligned}
& m\left(r, \varphi_{2}\right)=m\left(r, e^{\beta-\alpha} \cdot f\right) \leqq m(r, f)+m\left(r, e^{\beta-\alpha}\right) \\
& \leqq(2+o(1)) m\left(r, e^{\beta}\right)+(1+o(1)) m\left(r, e^{\beta}\right)=(3+o(1)) m\left(r, e^{3}\right) \\
& \quad\left(r \notin E^{\prime}, r \rightarrow \infty\right),
\end{aligned}
$$

and so

$$
\begin{aligned}
m\left(r, \varphi_{3}\right) & =m\left(r, 1-\varphi_{1}-\varphi_{2}\right) \leqq m\left(r, \varphi_{1}\right)+m\left(r, \varphi_{2}\right)+O(1) \\
& \leqq(4+o(1)) m\left(r, e^{\beta}\right) \quad\left(r \notin E^{\prime}, r \rightarrow \infty\right) .
\end{aligned}
$$

It follows from (4.14), (4.15), (4.17), (4.18) and (4.19) that

$$
\begin{array}{r}
m(r, \Delta)=O\left(\log r \cdot m\left(r, e^{\beta}\right)\right) \text { and } m\left(r, \Delta^{\prime}\right)=O\left(\log r \cdot m\left(r, e^{\beta}\right)\right) \\
\left(r \notin E^{\prime}, r \rightarrow \infty\right) .
\end{array}
$$

Substituting (4.16) and (4.20) into (4.17), we obtain 


$$
\begin{array}{r}
m\left(r, e^{\beta}\right)<O\left(\log r \cdot m\left(r, e^{\beta}\right)\right)+(6 k+o(1)) m\left(r, e^{\beta}\right)+O(\log r) \\
\left(r \notin E^{\prime}, r \rightarrow \infty\right) .
\end{array}
$$

Since $k<1 / 6$, (4.21) implies $m\left(r, e^{\beta}\right)=O(\log r)\left(r \in E^{\prime}, r \rightarrow \infty\right)$. This is a contradiction. Assume next that $\Delta \equiv 0$. This implies

$$
0 \equiv\left|\begin{array}{lll}
\varphi_{1} & \varphi_{2} & \varphi_{3} \\
\varphi_{1}^{\prime} & \varphi_{2}^{\prime} & \varphi_{3}^{\prime} \\
\varphi_{1}^{\prime \prime} & \varphi_{2}^{\prime \prime} & \varphi_{3}^{\prime \prime}
\end{array}\right|=\left|\begin{array}{ccc}
1 & \varphi_{2} & \varphi_{3} \\
0 & \varphi_{2}^{\prime} & \varphi_{3}^{\prime} \\
0 & \varphi_{2}^{\prime \prime} & \varphi_{3}^{\prime \prime}
\end{array}\right|=\left|\begin{array}{cc}
\varphi_{2}^{\prime} & \varphi_{3}^{\prime} \\
\varphi_{2}^{\prime \prime} & \varphi_{3}^{\prime \prime}
\end{array}\right| .
$$

Hence we have $\varphi_{2}=C \varphi_{3}+D$ (where $C$ and $D$ are constants.), i.e.

$$
-e^{\beta-\alpha} \cdot f=C f+D \text {. }
$$

If $f$ has a zero, (4.22) implies $D=0$, so $f \equiv 0 \equiv g$. If $f$ has no zeros, (4.22) implies $D=0$ and that $f=\frac{-D}{C+D e^{\beta-\alpha}}$. Since $f$ is entire, $c$ must vanish. Then $f=-D e^{r-, \beta}$. This and the fact that $\varphi_{1}+\varphi_{2}+\varphi_{3}=e^{\beta}-e^{\beta-\alpha} \cdot f+f \equiv 1$ imply

$$
e^{\beta}-D e^{\alpha-\beta}=1-D \text {. }
$$

Assume that $D=1$. In this case we have $f=-e^{\alpha-\beta}=-e^{\beta}$ and $g=e^{-\alpha} f=-e^{-\hat{\beta}+\alpha}$ $=-e^{-\beta}$. Hence $f g \equiv 1$. Assume that $D \neq 1$. In this case, using Lemma 5 , we obtain $2=\delta\left(0, e^{\beta}\right)+\delta\left(0, e^{\alpha-\beta}\right) \leqq 1$, which is impossible.

This completes the proof.

Remark. In the above Theorem 3, we consider the case of $\rho_{f}, o_{g}<\infty$. Then the same conclusion of Theorem 3 holds if we take the constant $k$ between zero and $1 / 4(0<k<1 / 4)$. To see this, we may refer to [6, Theorem 3]. This theorem and its proof show that $m(r, f) \sim m(r, g) \sim m\left(r, e^{\beta}\right)(r \rightarrow \infty)$ under our assumptions. Taking this fact into consideration, the reasoning in the above proof yields the fact stated as Remark. And we notice that this remark yields Theorem A.

5. Proof of Theorem 4. We refer to Ozawa's argument in [7, p. 416-p. 419]. In this case we have

$$
f=e^{\alpha} g, \quad f-1=e^{\beta}(g-1)
$$

with polynomials $\alpha$ and $\beta$.

Case 1. Assume that $e^{\beta}$ is a constant $c(\neq 0)$. Since $f$ has at least one zero, $f-1=c(g-1)$ implies $c=1$. Hence $f \equiv g$. This is a contradiction.

Case 2. Assume that $e^{\alpha-\beta}$ is a constant $c(\neq 0)$. If $c=1$, (5.1) implies $j \equiv g$, a contradiction. If $c \neq 1,(5.1)$ implies

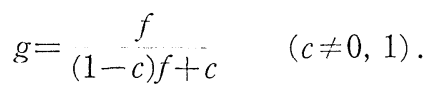

Since $g$ is an entire function, $(1-c) f+c=0$ has no roots. This yields $\delta(0, f)=0$. 
It follows from this, the assumption $(B)^{\prime}$, and Lemma 3 , that the genus of $f$ must be zero or one. So, if we put $f=P e^{r}$ with some canonical product $p$ and polynomial $\boldsymbol{r}$, the degree of $\gamma$ is at most one. This and Lemma 2 imply $m(r, f)$ $=o\left(r^{2}\right)(r \rightarrow \infty)$.

Now we set $f+c /(1-c)=e^{r}$ with some polynomial $\eta$. Since $m(r, f)=o\left(r^{2}\right)$ $(r \rightarrow \infty)$ and $f$ is not a constant, the degree of $\eta$ must be one : $\eta=a_{1} z+b_{1}(a \neq 0)$. Using the assumption $(B)^{\prime}$ and the fact that $\delta(0, f)=0$, we easily see that $a_{1}$ must be a purely imaginary number. So we have $|c /(1-c)|=e^{R e b_{1}}$. All the above results combine to show that $f=|c /(1-c)|\left(e^{\imath(a z+b)}-e^{2 d}\right)$ and $g=f /[(1-c) f+c]$ $(c(\neq 0,1)$ : a constant, $a(\neq 0), b, d$ : real constants $)$.

Case 3. Assume neither $e^{\beta}$ nor $e^{\alpha-\beta}$ are constants. From (5.1) we have

$$
f=\frac{1-e^{\beta}}{1-e^{\beta-\alpha}} .
$$

Since $f$ is an entire function, we deduce from (5.3)

$$
\beta(z)=2 \pi \imath L(y(z)), \quad 2 \pi \imath y(z)=\beta(z)-\alpha(z)
$$

with a polynomial $L(z)$ of degree $\geqq 1$ (See [f, Theorem 3.]).

Now we assert that the degree of $L$ must be one. Suppose contrary to this assertion. Then simple computations give

$$
m\left(r, e^{\beta}\right) \sim m(r, f) \quad(r \rightarrow \infty) \text { and } \delta(0, f)=0 .
$$

Using (5.5) and the assumption (B)', Lemma 3 implies

$$
m\left(r, e^{\beta}\right)=o\left(r^{2}\right) \quad(r \rightarrow \infty) .
$$

On the other hand, since the degree of $y(z)$ in (5.4) is not less than one, the degree of $\beta(z)$ in (5.4) is not less than two, so we deduce that

$$
m\left(r, e^{\beta}\right) \geqq O\left(r^{2}\right) \quad(r \rightarrow \infty) .
$$

(5.6) and (5.7) evidently contradict each other. Hence the degree of $L$ must be one.

Assume that the degree of $L$ is one. Then $f(z)$ must be of the following form :

$$
f(z)=\frac{1-e^{2 \pi \imath m y(z)}}{1-e^{2 \pi \imath y(z)}} \quad(m: \text { an integer })
$$

(See [6, Theorem 3]). First assume that $|m| \geqq 2$. In this case, the roots of $f=0$ are the ones of $y(z)=n /|m| \neq$ an integer ( $n:$ an integer). We deduce from this and the assumption $(\mathrm{B})^{\prime}$ that the degree of $y(z)$ must be one : $y(z)=$ $a z+b(a \neq 0)$. Here $a$ and $b$ are clearly real. Substituting $y(z)=a z+b$ into (5.4), we obtain

$$
\beta(z)-\alpha(z)=2 \pi i(a z+b) .
$$

Comparing (5.3) with (5.8) and using (5.1), we have 
and hence

$$
\begin{aligned}
\beta(z) & =m(\beta(z)-\alpha(z))+2 \pi i l \quad(l: \text { an integer }) \\
& =2 \pi \imath m(a z+b)+2 \pi i l
\end{aligned}
$$

$$
\alpha(z)=2 \pi i(m-1)(a z+b)+2 \pi i l .
$$

Thus we obtain from (5.8), (5.9), (5.10) and (5.1)

$$
f(z)=\frac{1-e^{2 \pi \imath m(a z+b)}}{1-e^{2 \pi i(a z+b)}}, \quad g(z)=\frac{e^{-2 \pi \imath(m-1)(a z+b)}-e^{2 \pi l(a z+b)}}{1-e^{2 \pi i(a z+b)}} .
$$

Finally we consider the case of $m=-1,0$ and 1 . Assume that $m=-1$. Then from (5.8) we have $f=-e^{-2 \pi \imath y(z)} \neq 0$, a contradiction. Assume that $m=0$. Then from (5.8) we have $f \equiv 0$. This is absurd. Assume that $m=1$. Then from (5.8) we have $f \equiv 1$, which is untenable.

This completes the proof.

6. Proof of Theorem 5. Firstly, making use of Theorem B, we have $\delta(0, f)$ $=0$. Then the assumption (B)' implies that

$$
\text { the genus of } f \leqq 1 \text { and } m(r, f)=o\left(r^{2}\right) \quad(r \rightarrow \infty) \text {. }
$$

Hence, in view of Lemma 4, we have

$$
\begin{aligned}
m(r, g) & \leqq N(r, 0, g)+N(r, 1, g)+O(\log r)=N(r, 1, f)+O(\log r) \\
& \leqq m(r, f)+O(\log r)=o\left(r^{2}\right) \quad(r \rightarrow \infty) .
\end{aligned}
$$

Consequently the assumptions $(\mathrm{A})^{\prime}$ and $(\mathrm{B})^{\prime}$ imply

$$
g(z)=e^{a z} \quad(a \neq 0) .
$$

Now, from $\rho_{f}, \rho_{g}<\infty$ and the assumption (A)' we obtain

$$
f(z)=1+(g(z)-1) e^{\alpha(z)} .
$$

with a polynomial $\alpha$. (6.1), (6.2) and (6.3) imply that the degree of $\alpha$ must be zero or one: $\alpha(z)=c z+d((c, d) \neq(0,0))$. Therefore we have

$$
f(z)=1+e^{(a+c) z+d}-e^{c z+d} \quad(a(\neq 0),(c, d) \neq(0,0))
$$

Next we assert that both $a$ and $c$ are purely imaginary numbers. First we note that (6.2) implies

$$
n(r, 1, g) \sim \frac{|a|}{\pi} r, \quad N(r, 1, g) \sim \frac{|a|}{\pi} r \quad(r \rightarrow \infty) .
$$

Then, by the first fundamental theorem, we obtain

$$
m(r, f) \geqq N(r, 1, f)+O(1)=N(r, 1, g)+O(1) \quad(r \rightarrow \infty) .
$$

It follows from (6.1) and (6.6) that the genus of $f$ must be one. Hence by Lemma 3 , we see that the number of the roots of $f=0$ which lie on the posi- 
tive real axis is infinite, and that the same is true for the zeros of $f$ on the negative real axis. From this we easily deduce that both $a$ and $c$ are purely imaginary numbers. Therefore $f$ must be of the following form:

$$
\begin{aligned}
& f(z)=1+|c| e^{i[(\alpha+\beta) z+i]}-|c| e^{2(\beta z+i)}, \\
& c=|c| e^{2 i} \neq 0, \quad \alpha, \beta, \gamma: \text { real constants. }
\end{aligned}
$$

Case 1. Assume that $f$ is a real entire function. In this case we have $\sin ([\alpha+\beta] x+\gamma)-\sin (\beta x+\gamma)=0$ for any real $x$. This implies that $\alpha+2 \beta=0$, $\gamma=(m+1 / 2) \pi(m:$ an integer $)$ and $c= \pm i|c|$. Substituting these into (6.7) we have $f(z)=1 \pm i|c| e^{-\imath \beta z} \mp i|c| e^{\imath \beta z}(|c| \neq 0, \beta=0)$. Here using the assumption (B)', we have $|c| \geqq 1 / 2$.

Case 2. Assume that $f$ is a non-real entire function. Since the roots of $g=1$ are $\{2 n \pi \imath / a\}_{n=-\infty}^{+\infty}$ and $a$ is purely imaginary number, we see that all the roots of $f=1$ lie on the real axis. This and the assumption $(B)^{\prime}$ imply $f \in A_{A}$. Hence we can apply Lemma 1 to $f$. Firstly we rewrite the functions (i) and (ii) in Lemma 1 in the following forms.

$$
\begin{cases}\text { (1) } f(z)=\frac{e^{\imath\left(\eta-\eta_{1}\right)}}{2 \imath \sin \left(\eta-\eta_{1}\right)}\left[e^{2 \imath\left(\xi z+\eta_{1}\right)}-1\right], \\ \text { (ii) } f(z)= \begin{cases}1+e^{2 \imath(\bar{\xi} z+\eta)}+\cdots+e^{2 \imath(p-1)\left(\xi z+\eta_{1}\right)} & (p=2,3, \cdots), \\ -e^{-2 \imath(\xi z+\eta)}-\cdots-e^{2 \imath p\left(\xi z+\eta_{1}\right)} & (p=-1,-2, \cdots) .\end{cases} \end{cases}
$$

Case 2.1. Assume that both $\alpha$ and $\beta$ are positive in (6.7). Comparing (6.7) with (ii) (the case of $p=2)$ in (6.8), we deduce that $|c|=1,(\beta z+\gamma)+(2 k+1) \pi=$ $2(\xi z+\eta)(k:$ an integer) and that $([\alpha+\beta] z+\gamma)+2 m \pi=4(\xi z+\eta)(m:$ an integer $)$. These imply $\alpha=\beta, \quad \gamma=2 l \pi$ (l: an integer) and $c=1$. Thus we have $f(z)==$ $1+e^{2 \imath \alpha z}-e^{2 \alpha z}(\alpha \neq 0)$.

Case 2.2. Assume that $\alpha<0$ and $\beta>0$ in (6.7). Comparing (6.7) with (6.8), we deduce that $\alpha+\beta \geqq 0$. If $\alpha+\beta=0$, we use (6.7) to obtain $f(z)=1+c-c e^{i \beta z}$. However, the assumption $(\mathrm{B})^{\prime}$ requires that $c=-1 / 2+i b$ (b: a real constant). If $\alpha+\beta>0$, we deduce from (6.7) and (ii) in (6.8) that $|c|=1,([\alpha+\beta] z+i)+2 m \pi=$ $2(\xi z+\eta)$, and that $(\beta z+\gamma)+(2 k+1) \pi=4(\xi z+\eta)$. These imply that $\alpha==-\beta / 2, \quad \gamma==$ $(2 l+1) \pi$ pnd $c=-1$. Hence we have $f(z)=1-e^{\imath(\beta / 2) z}+e^{2 \beta z}$.

Case 2.3. Assume that $\alpha>0$ and $\beta<0$ in (6.7). Doing in the same way we deduce that $\alpha+\beta=0$. Hence we have the same result as in the first half of Case 2.2.

Case 2.4. Assume that both $\alpha$ and $\beta$ are negative in (6.7). Doing in the same way, we have the same result as in Case 2.1.

Case 2.5. Assume finally that $\beta=0$ in (6.7). In this case, from (6.7) we have $f(z)=1-c+c e^{\imath \alpha z}$. Here using the assumption $(\mathrm{B})^{\prime}$, we have $c=1 / 2+i b(b$ : a real constant)

This completes the proof. 


\section{REFERENCES}

[1] A. EDREI, Meromorphic functions with three radially distributed values, Trans. Amer. Math. Soc. 78 (1955) 276-293.

[2: A. Edrei ,W. H. J. Fucis and S. Hellerstein, Radial distribution and deficiencies of the values of a meromorphic function, Pacific. J. Math. 11, (1961) 135151.

[3] W. K. HaYmax, Meromorphic functions, Oxford, (1964).

[4] R. Neva laivisa, Le théorème de Picard-Borel et la théorie des fonctions meromorphes, Paris, Gauthier-Villars, (1929).

[5] K. Nino aNd M. Ozawa, Deficiencies of an entıre algebroid function, Kōdai. Math. Sem. Rep. 22, (1970) 98-113.

[6] C.F. Osgood And C.C. YANG, On the quotient of two integral functions, J. Math. Anal. Appl. 54 (1976) no 2, 408-418.

[7] M. Ozawa, Unicity theorems for entire functions, J. d'Analyse Math. Vol. 30, (1976) $411-420$.

Department of Mathematics,

Tokyo Institute of TEChNOLOGY.

Current Adress

Department of Mathematics,

Daido Institute of TeChNology,

2-21 Daido-Cho, Minami-Ku,

NAGOYA, JAPAN 